\title{
Echocardiographic strain
}

\author{
Sofia Prieto MD, Omid Hosseini BS, Pooja Sethi MD
}

\begin{abstract}
Echocardiography is a readily available imaging modality used to evaluate cardiac function. Recently, the addition of strain measurements has provided additional information by evaluating the intrinsic myocardial function and detecting subclinical systolic dysfunction. As with any emerging technique or application, it is imperative to understand how the information gathered in the study can be applied to clinical practice. This review article examines how echocardiographic strain analysis can be applied to clinical practice and its use as a promising prognostic resource in many cardiovascular conditions.
\end{abstract}

Keywords: echocardiographic strain, left ventricular dysfunction, speckle tracking, myocardial dysfunction

Echocardiography is a routinely used image-based diagnostic method for evaluating cardiac function. Parameters, primarily left ventricular ejection fraction, are used daily for diagnosis, treatment, and prognosis of cardiac diseases in clinical practice. Although conventional echocardiography is considered reliable, the subjective estimation of wall motion through calculation and observer variability may not always provide accurate information about myocardial properties. ${ }^{1,2}$ In recent years, strain echocardiography has been increasingly used in clinical practice. ${ }^{2}$ This method allows clinicians to evaluate myocardial function that is not visually assessable and provides an accurate and comprehensive evaluation of intrinsic myocardial function that may have multiple clinical applications. ${ }^{3}$

Strain is a dimensionless measurement of deformation. Three different forms of myocardial deformation can be evaluated: radial strain represents the myocardial thickening in a short-axis plane; circumferential strain represents myocardial shortening in a short-axis plane; and longitudinal strain represents the myocardial shortening in the long-axis plane. ${ }^{4}$ Strain is

Corresponding author: Sofia Prieto

Contact Information: Sofia.Kennedy@ttuhsc.edu DOI: 10.12746/swrccc.v8i36.767 an index which indicates a change in myocardial muscle dimensions. It is derived from the formula: (L1-L0)/ $\mathrm{L} 0 \times 100 \%$. If myocardial contractile units shorten, the number is a negative. If myocardial contractile units thicken, the number is positive. Tissue Doppler imaging (TDI) and two-dimensional speckle tracking are the echocardiographic techniques used to measure strain and strain rate. Tissue Doppler imaging depicts myocardial motion, and strain is derived from velocity data. ${ }^{2}$ Two-dimensional speckle tracking uses ultrasonic speckles to trace movement in different planes.

With emerging techniques, definitions, and applications, the need to standardize deformation imaging is crucial for clinical practice. ${ }^{5}$ The European Association of Cardiovascular Imaging and the American Society of Echocardiography (ASE) have produced consensus definitions, calculations, and procedures related to deformation imaging. ${ }^{5}$ Left ventricular global strain varies with age, sex, and ventricular loading conditions, and based on ASE criteria, a value less than (more negative) than $-18 \%$ is considered normal. ${ }^{6}$ Numbers greater than a $-15 \%$ are abnormal. By adopting this standard, strain echocardiography can provide important diagnostic and prognostic information in the clinical setting.

Echocardiographic strain analysis is now being used more routinely in clinical practice due to its 
reproducibility and feasibility. It can detect subclinical systolic dysfunction even when left ventricular systolic function is preserved. Global strain can provide prognostic value for patients with any ejection fraction, reduced or preserved, whereas left ventricular ejection fraction loses its prognostic implications in individuals with an ejection fraction greater than $40 \% .{ }^{7} \mathrm{~A}$ reduced global longitudinal strain has correlated with an increase in mortality, even in patients with normal ejection fractions.

Clinical applications of two-dimensional speckle tracking echocardiography derived strain can help evaluate cardiomyopathies and heart failure. A dilated cardiomyopathy has a reduced strain in all three planes: longitudinal, radial and circumferential. ${ }^{2}$ Nahum et al. reported that chronic heart failure patients with dilated cardiomyopathy had reduced global strain and poor long-term prognosis. ${ }^{8}$ Another study published in 2018 found that global strain was independently predictive of mortality in acute heart failure patients while acute change in ejection fraction did not correspond to overall mortality. ${ }^{7}$ In heart failure patients with preserved ejection fraction, global strain was reduced compared to age- and gender-matched controls. ${ }^{2}$ Furthermore, in these patients, reduced global strain was associated with increased risk of cardiovascular death, heart failure hospitalizations, and cardiac arrests.

Global strain can be useful in differentiating physiological left ventricular hypertrophy from various pathological forms of hypertrophic cardiomyopathy. ${ }^{2}$ For example, athlete's heart is due to adaptive cardiac changes that occur over time with training and, therefore, is not considered pathological. Global strain is not impaired in these individuals. Global strain is reduced in patients with hypertrophic cardiomyopathy, with hypertrophy related to hypertension, and in cardiac amyloidosis. Sun et al. found that a global strain of about $-15 \%$ to $-18 \%$ in patients with hypertrophic cardiomyopathy, conferred better survival. ${ }^{9}$ While patterns and distribution of strain anomalies differ by type of cardiomyopathy, all appear to have a reduction in strain that correlates with worse overall prognosis and clinical course.

Several studies have investigated the use of echocardiographic strain as a predictor for chemotherapyrelated cardiotoxicity. These studies have shown that global longitudinal strain could be used in oncology patients to monitor for and possibly predict cardiotoxicity. Mousavi et al. recently evaluated 450 patients with hematological malignancy before and after chemotherapy for cardiac events. They found that patients with a global stain value greater than $-17.5 \%$ and left ventricular ejection fraction of $50-59 \%$ prior to receiving cardiotoxic chemotherapy were up to six times more likely to develop symptomatic heart failure and cardiac death. ${ }^{10} \mathrm{~A}$ relative reduction in global longitudinal strain greater than $15 \%$ compared from baseline is the threshold defined by the ASE to identify subclinical left ventricular dysfunction in patients receiving chemotherapy. ${ }^{11}$

Other areas in which global longitudinal strain provides utility include evaluation of coronary artery disease revascularization and recovery. Patients with coronary artery disease have significantly lower global strain values. ${ }^{12}$ Studies have reported a reduction in global strain with acute myocardial infarction and a normalizing of strain pattern back to baseline after treatment of the infarction. This implies that strain could be a used as a predictor of cardiac remodeling.

The evaluation of ventricular function using speckle tracking strain provides significant prognostic information for many cardiovascular conditions. It is highly sensitive in recognizing subclinical left ventricular dysfunction which may help guide early therapy by providing risk stratification and improve prognostic evaluation. While it is imperative that standardized protocols become established, the utility of this technique is promising, and it should be considered when conducting a thorough cardiovascular assessment of patients.

Article citation: Prieto S, Hosseini O, Sethi P. Echocardiographic strain. The Southwest Respiratory and Critical Care Chronicles 2020;8(36)58-60

From: Department of Internal Medicine, Texas Tech University Health Sciences Center, Lubbock, Texas

Submitted: 9/29/2020

Accepted: 10/6/2020

Reviewer: Scott Shurmur MD Conflicts of interest: none This work is licensed under a Creative Commons Attribution-ShareAlike 4.0 International License. 


\section{REFERENCES}

1. Park JH. Two-dimensional echocardiographic assessment of myocardial strain: important echocardiographic parameter readily useful in clinical field. Korean Circ J 2019;49(10): 908-931.

2. Trivedi S,Altman M, Stanton T, et al. Echocardiographic strain in clinical practice. Heart Lung Circ 2019;28:1320-1330

3. Dandel M, Lehmkuhl H, Knosalla C, et al. Strain and strain rate imaging by echocardiography-basic concepts and clinical applicability. Curr Cardiol Rev 2009;5(2):133-148.

4. Delgado V, Ypenburg C, van Bommel RJ, et al. Assessment of left ventricular dyssynchrony by speckle tracking strain imaging comparison between longitudinal, circumferential, and radial strain in cardiac resynchronization therapy. J Am Coll Cardiol 2008;51(20):1944-52.

5. Voigt JU, Pedrizzetti G, Lysyansky P, et al. Definitions for a common standard for 2D speckle tracking echocardiography: consensus document of the EACVI/ASE/Industry Task Force to standardize deformation imaging. Eur Heart J Cardiovasc Imaging 2015;16(1):1-11.

6. Collier P, Phelan D, Klein A. A Text in context: myocardial strain measured by speckle-tracking echocardiography. J Am Coll Cardiol 2017;69(8):1043-56.
7. Park JJ, Park JB, Park JH, et al. Global longitudinal strain to predict mortality in patients with acute heart failure. J Am Coll Cardiol 2018;71(18):1947-57.

8. Nahum J, Bensaid A, Dissault C, et al. Impact of longitudinal myocardial deformation on the prognosis of chronic heart failure patients. Circ Cardiovasc Imaging 2010;3(3):249-56.

9. Sun JP, Stewart WJ, Yang XS, et al. Differentiation of hypertrophic cardiomyopathy and cardiac amyloidosis from other causes of ventricular wall thickening by two-dimensional strain imaging echocardiography. Am J Cardiol 2009;103(3):411-5.

10. Ali MT, Yucel E, Bouras S, et al. Myocardial strain is associated with adverse clinical cardiac events in patients treated with anthracyclines. J Am Soc Echocardiogr 2016;29(6): 522-7.

11. Plana JC, Galderisi M, Barac A, et al. Expert consensus for multimodality imaging evaluation of adult patients during and after Cancer therapy: a report from the American Society of Echocardiography and the European Association of Cardiovascular Imaging. J Am Soc Echocardiogr 2014;27(9): 911-39.

12. Hung C, Verma A, Uno H, et al. Longitudinal and circumferential strain rate, left ventricular remodeling and prognosis after myocardial infarction. J Am Coll Cardiol 2010; 56(22):1812-22. 